\title{
Estresse ocupacional e saúde mental no trabalho em saúde: desigualdades de gênero e raça
}

\section{Occupational stress and mental health in healthcare work: inequalities of gender and race}

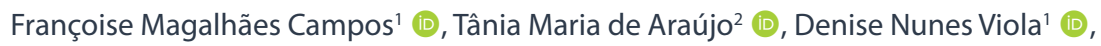 \\ Paula Caroline Santos Oliveira² (D), Camila Carvalho de Sousa² (D) \\ 'Programa de Pós-graduação em Saúde Ambiente e Trabalho, Faculdade de Medicina, Universidade Federal da Bahia (UFBA) - \\ Salvador (BA), Brasil. \\ ${ }^{2}$ Programa de Pós-graduação em Saúde Coletiva, Universidade Estadual de Feira de Santana (UEFS) - Feira de Santana (BA), Brasil.
}

\begin{abstract}
Como citar: Campos FM, Araújo TM, Viola DN, Oliveira PCS, Sousa CC. Estresse ocupacional e saúde mental no trabalho em saúde: desigualdades de gênero e raça. Cad Saúde Colet, 22020;28(4):579-589. https://doi.org/10.1590/1414$462 \times 202028040559$
\end{abstract}

\begin{abstract}
Resumo
Introdução: Os aspectos psicossociais do trabalho são fontes de estresse ocupacional, com impactos na saúde mental. Esta relação pode ser determinada pelo gênero e pela raça/cor da pele. Objetivo: Avaliar associação entre estressores ocupacionais e saúde mental, focalizando desigualdades de gênero e raça/cor da pele entre trabalhadores da saúde. Método: Estudo transversal com 3.084 trabalhadores de saúde da Bahia. Estressores ocupacionais foram avaliados pelo modelo demanda-controle (MDC), utilizando o Job Content Questionnaire. A variável desfecho, transtornos mentais comuns (TMC), foi avaliada pelo SRQ-20. Conduziuse análise descritiva, bivariada e multivariada, estratificada por gênero e raça/cor da pele. Resultados: A prevalência de TMC foi maior entre as mulheres (negras: 23,7\%, e não negras: 19,6\%), quando comparada com a verificada entre os homens (negros: 17,6\%, e não negros: 14,7\%). Observou-se associação de TMC com todos os grupos do MDC, para as mulheres negras, e com trabalho ativo e de alta exigência entre mulheres não negras. Entre os homens, a associação não foi estatisticamente significante. Conclusão: Observaram-se diferenciais de gênero e raça/cor da pele na ocorrência de TMC e na associação com estressores ocupacionais, com prevalências mais elevadas entre as mulheres, principalmente as mulheres negras.
\end{abstract}

Palavras-chave: aspectos psicossociais no trabalho; transtorno mental comum; gênero; raça; trabalhadores de saúde.

\begin{abstract}
Background:The psychosocial aspects of work are sources of occupational stress, with impacts on mental health. This relationship can be determined by gender and race/skin color. Objective: To evaluate the association between occupational stressors and mental health, focusing on gender and race/skin color inequalities among healthcare workers. Method: Cross-sectional study with 3,084 healthcare workers from Bahia. Occupational stressors were assessed using the demand-control (CDM) model, using the Job Content Questionnaire. The outcome variable, common mental disorders (CDM), was assessed by the SRQ-20. A descriptive, bivariate, and multivariate analysis was carried out, stratified by gender and race/skin color. Results: The prevalence of CMD was higher among women (black: $23.7 \%$, and non-black: $19.6 \%$ ) than among men (blacks: $17.6 \%$, and non-blacks: $14.7 \%$ ). There was an association of CMD with all groups of the MDC, for black women, and with active and highly demanding work among non-black women. Among men, the association was not statistically significant. Conclusion: Differences in gender and race/skin color were observed in the occurrence of CMD and in the association with occupational stressors, with higher prevalence among women, especially black women.
\end{abstract}

Keywords: psychosocial aspects at work; common mental disorder; gender; race; health workers.

Este é um artigo publicado em acesso aberto (Open Access) sob a licença Creative Commons Attribution, que permite uso, distribuição e reprodução em qualquer meio, sem restrições desde que o trabalho original seja corretamente citado.

\footnotetext{
Trabalho realizado em cinco municípios baianos (Feira de Santana, Salvador, Jequié, Santo Antônio de Jesus e Itabuna), com trabalhadores de saúde da Atenção Básica e da Média Complexidade - Feira de Santana (Bahia), Brasil.

Correspondência: Camila Carvalho de Sousa. E-mail: camilacarvalho255@gmail.com

Fonte de financiamento: FAPESB.

Conflito de interesses: nada a declarar.

Recebido em: Nov. 16, 2018. Aceito em: Out. 20, 2019
} 


\section{INTRODUÇÃO}

A ocorrência de doenças no ambiente laboral pode ser influenciada por fatores socioeconômicos, individuais e hábitos de vida e do trabalho. Dentre esses fatores, destacamse o gênero e a raça. Diferenças nessas características podem estruturar desigualdades nas condições e características do trabalho, que, por sua vez, podem produzir ou agravar diversos tipos de problemas de saúde física e mental, como os transtornos mentais ${ }^{1,2}$.

Tanto raça quanto gênero são fatores reconhecidos como marcadores sociais ${ }^{3,4}$. A análise do papel dessas desigualdades é relevante para uma melhor compreensão das vulnerabilidades que se tecem nos contextos laborais, podendo contribuir para orientação e estímulo de políticas de promoção e proteção à saúde. Nesta perspectiva, este estudo, embora tenha como foco a relação entre estressores ocupacionais e a saúde mental, realiza a análise à luz de potenciais desigualdades entre homens e mulheres, brancos/as e negros/as.

Estressores ocupacionais destacam-se como potenciais determinantes para a ocorrência dos transtornos mentais comuns $(T M C)^{5}$. Os TMC correspondem a sinais e sintomas psicossomáticos, como: fadiga, insônia, irritabilidade, dificuldade de concentração e esquecimento, constituindo-se importante problema de saúde pública, com impactos em diversas esferas da vida social e pessoal, e envolvendo elevados custos econômicos e sociais ${ }^{6}$.

A análise de estressores ocupacionais tem sido feita com a utilização de diversos modelos teóricos e instrumentos de mensuração. O modelo demanda-controle (MDC), proposto por Karasek (1979), avalia o estresse ocupacional a partir das dimensões: controle sobre o próprio trabalho e demanda psicológica envolvida na atividade laboral. Sugere-se que o adoecimento mental se relaciona centralmente a essas dimensões do trabalho, as quais, quando combinadas, geram diferentes situações laborais com impacto diferenciado na saúde dos trabalhadores ${ }^{7}$. Neste estudo avaliou-se esse pressuposto do modelo.

Como já mencionado, as desigualdades raciais e de gênero, ao estabelecerem condições de trabalho e de vida diferenciadas, influenciam a saúde mental dos trabalhadores. Apesar de certo reconhecimento das influências de gênero e raça na situação de saúde das pessoas ${ }^{8}$, a literatura evidencia carência de estudos com este foco, especialmente no campo de saúde e trabalho. Essa lacuna dificulta a identificação das necessidades dos grupos mais vulneráveis. Assim, explorar esta perspectiva analítica pode contribuir para compreender como condições sociais atuam para ampliar ou reduzir processos de adoecimento em contextos laborais 9 .

Este estudo tem como objetivo principal avaliar a associação entre estresse ocupacional e saúde mental, focalizando possíveis desigualdades de gênero e raça, entre trabalhadores/ as de saúde. Adicionalmente, considerando a escassez de estudos ocupacionais abordando diferenciais de gênero e raça no Brasil, este estudo também descreve as características de interesse segundo os grupos de gênero e raça, com a finalidade de estabelecer um perfil da população estudada, nessa perspectiva.

Espera-se estimular a elaboração de medidas para proteger ou reduzir danos à saúde mental, com base em melhorias nas condições de trabalho, sensibilização dos gestores e estímulo para avanços em políticas públicas, considerando-se processos de proteção à saúde dos grupos mais vulneráveis.

\section{MÉTODO}

Estudo transversal, realizado entre 2011 e 2012, com 3.084 trabalhadores/as da atenção básica e de média complexidade de cinco municípios baianos. A amostra foi definida em etapas sucessivas: a) obteve-se a lista nominal de todos os trabalhadores em atividade nos serviços de saúde da atenção básica e de média complexidade; b) estimou-se o tamanho amostral com base no total da população e em diferentes desfechos de saúde; c) definido o tamanho amostral, estratificou-se a amostra por três níveis: área geográfica, nível de assistência - atenção básica e média complexidade - e grupo ocupacional. A composição da amostra foi definida com base na participação percentual de cada grupo por nível de estratificação estabelecida; d) estimado o tamanho amostral em cada estrato, foram sorteados os trabalhadores para comporem a amostra com base em listagem de números aleatórios do Epilnfo 6.04d. Após o 
sorteio, os trabalhadores foram contatados nos próprios locais de trabalho, sendo realizadas até três visitas para que a entrevista fosse realizada.

Os dados foram coletados por meio de questionário autoaplicável, respeitando-se as normas e recomendações da Resolução n $466 / 12$ do Conselho Nacional de Saúde. O projeto foi aprovado pelo Comitê de Ética em Pesquisa sob o protocolo de número: CAAE 0086.0.059.000091.

A variável desfecho foi a presença de TMC, avaliada pelo Self-Reporting Questionnaire (SRQ20), instrumento de triagem de transtornos mentais, composto por 20 questões dicotômicas (sim/não), que englobam sintomas somáticos, depressivos e de ansiedade nos últimos 30 dias anteriores ao questionário. Para definição de suspeição de TMC, computaram-se as questões respondidas positivamente com valor superior ou igual ao ponto de corte adotado (7 pontos para mulheres e 5 pontos para homens) ${ }^{10}$.

A variável de exposição, estresse ocupacional, foi mensurada pelo Job Content Questionnaire (JCQ). Este instrumento avalia estressores ocupacionais, mensurando as dimensões de controle sobre o trabalho (9 itens) e as demandas psicológicas (5 itens). As situações de trabalho propostas pelo MDC foram construídas utilizando a mediana como ponto de corte para definição das categorias (alto/a e baixo/a) ${ }^{11}$. Em sequência, foram combinadas situações de trabalho previstas no modelo: alta exigência (alta demanda e baixo controle), trabalho ativo (alta demanda e alto controle), trabalho passivo (baixa demanda e baixo controle) e baixa exigência (baixa demanda e alto controle). Para a análise, o grupo referência (não exposto) adotado foi o de baixa exigência. O grupo de alta exigência foi considerado de maior exposição, como recomendado em outros estudos ${ }^{12}$.

As covariáveis foram: características sociodemográficas (idade, filhos, situação conjugal, nível de escolaridade), informações gerais sobre o seu trabalho (categoria profissional, tempo de trabalho em anos, vínculo de trabalho atual, jornada semanal, turno de trabalho, outros vínculos profissionais); apoio social, hábitos de vida (lazer e atividade física) e sobrecarga doméstica - indicador construído somando as tarefas domésticas básicas, multiplicando pelo número de moradores $(S D=(\text { lavar + passar + limpar + cozinhar }) \times(m-1))^{13}$.

Para análise, utilizaram-se os programas STATA e o pacote $\mathrm{R}$ comander do software $\mathrm{R}$. As análises foram estratificadas por gênero e raça. A categoria raça foi respondida de acordo com o Instituto Brasileiro de Geografia e Estatística (IBGE) para o censo nacional: branca, preta, parda, amarela e indígena. As respostas preta e parda foram agrupadas, formando os grupos de negros/as, e as demais categorias constituíram o grupo de não negros/as. Na análise, foram criados os seguintes estratos: homens negros, homens não negros, mulheres negras, mulheres não negras.

Realizou-se análise descritiva por gênero e raça, com o intuito de caracterizar a população de estudo, mensurar o estresse laboral e a ocorrência de TMC. Na análise bivariada, foram calculadas as prevalências $(\mathrm{P})$ e razões de prevalências (RP). $\mathrm{O}$ teste qui-quadrado foi utilizado para análise de significância estatística, considerando-se nível de significância de 5\%.

Para avaliação simultânea das variáveis de interesse, empregou-se análise de regressão logística múltipla (ARLM). A ARLM foi utilizada em função de a variável desfecho tratar-se de uma variável dicotômica, TMC (presente, ausente). Para a pré-seleção das variáveis de entrada no modelo multivariado, utilizaram-se, como critério de retenção das variáveis, valores de $p \leq 0,17^{14}$. Na seleção do modelo final, adotou-se critério de retenção de $p<0,05$. As variáveis que mesmo não apresentando valor de $p$ significante, mas que, segundo a literatura, tinham importância para a ocorrência do desfecho, permaneceram no modelo final Obtido o modelo final, as razões de prevalência (RP) e os intervalos de confiança de $95 \%$ foram obtidos por Regressão de Poisson com variação robusta ${ }^{15}$. O diagnóstico do desempenho do modelo final foi avaliado por meio da Curva ROC.

\section{RESULTADOS}

Entre os/as 3.084 trabalhadores/as que participaram do estudo, a maioria $(78,3 \%)$ era do sexo feminino e se autodeclararam raça negra $(80,6 \%)$. Do total estudado, $503(16,6 \%)$ eram 
homens negros, $155(5,1 \%)$ homens não negros, 1.938 (64,0\%) mulheres negras e 432 (14,3\%) mulheres não negras.

Evidenciou-se predomínio de trabalhadores jovens, com até 40 anos de idade. Os homens não negros apresentaram maior percentual de nível de escolaridade superior $(68,7 \%)$, seguidos pelas mulheres não negras $(57,4 \%)$. Situação inversa foi observada na população negra, com predomínio de quem não possuía ensino superior: $61,5 \%$, entre as mulheres, e $58,9 \%$, entre os homens. A maioria dos/as entrevistados/as afirmou ter companheiro/a e possuir filhos.

Com relação à ocupação, observou-se que, entre os homens, a categoria de maior percentual foi a de "outras profissões" que englobava as profissões do setor administrativo, técnico de nível médio, serviços gerais e vigilantes (41,5\% negros e 31,4\% não negros). Entre os homens negros, a segunda maior frequência foi observada na categoria de agentes de saúde (ACS/ACE) (38,1\%); já entre os homens não negros, foi a profissão de médico (22,2\%).

Entre as mulheres, predominaram as categorias de agente de saúde (ACS/ACE) (41,9\% negras, $24,9 \%$ não negras), seguida da categoria outras profissões (28,3\% negras, $22,8 \%$ não negras). Registra-se que as categorias profissionais de nível médio foram mais relatadas entre a população negra, já as categorias de nível superior foram referidas com maior frequência entre não negros/as.

Dentre os grupos, a maioria relatou praticar atividades regulares de lazer. No entanto, observou-se maior percentual de ausência de atividades de lazer entre as mulheres (negras: 17,6\%, não negras: $19,6 \%$ ). A realização de atividades físicas, obteve maior percentual entre os homens não negros $(65,2 \%)$ seguido dos homens negros $(56,5 \%)$. Entre as mulheres, predominou a não realização de atividades físicas (62,6\% negras, 51,6\% não negras).

A responsabilidade pelas atividades domésticas foi maior entre as mulheres, quando comparada a essa responsabilidade entre os homens, destacando-se entre as mulheres negras $(60,7 \%)$, sendo este grupo também o de maior percentual de sobrecarga doméstica alta (54,7\%).

O apoio social no trabalho também apresentou diferenças expressivas entre homens e mulheres. Entre os homens, predominou alto apoio, enquanto, entre as mulheres, a situação foi inversa, com maiores percentuais em baixo apoio entre as negras $(71,7 \%)$ e entre as não negras $(69,3 \%)$.

A demanda psicológica alta foi observada em maiores percentuais entre homens e mulheres não negros/as, sendo $56,1 \%$ e $51,0 \%$, respectivamente. No entanto, o baixo controle sobre o próprio trabalho foi mais presente entre os/as negros/as, sendo 49,3\% entre homens negros e $51,6 \%$ entre mulheres negras.

Com relação aos grupos do MDC, destaca-se o maior percentual de alta exigência entre as mulheres negras $(24,7)$ e menor percentual entre os homens não negros $(17,0 \%)$ (Tabela 1$)$.

As mulheres negras apresentaram maior prevalência de TMC (23,7\%), seguidas pelas não negras (19,6\%). A prevalência de TMC entre os homens foi menor, alcançando o menor patamar entre homens não negros (14,7\%) (Figura 1).

Na análise de associações brutas, os TMC associaram-se à elevada demanda psicológica entre homens negros e mulheres (negras e não negras), e ao baixo controle entre as mulheres (negras e não negras) (Tabela 2).

Tanto entre os homens quanto entre as mulheres, observou-se associação estatisticamente significante entre TMC e alta exigência no trabalho. Destacam-se associações mais robustas entre as mulheres, especialmente entre as mulheres negras (Tabela 2).

No modelo final de análise, com avaliação simultânea das variáveis de interesse, foram encontradas diferenças relevantes na associação entre TMC e categorias do modelo demandacontrole (MDC): entre as mulheres negras, observou-se associação para todos os grupos comparados com a baixa exigência, tendo sido ajustado por vínculo de trabalho (Tabela 3). Entre as mulheres não negras, os TMC mantiveram-se associados à situação de alta exigência e trabalho ativo ajustado por ter filhos, tipo de vínculo de trabalho e sobrecarga doméstica.

Embora, no modelo final de análise de TMC e estressores ocupacionais, entre os homens, a associação não tenha sido significante, cabe o registro de que as razões de prevalência para o trabalho em alta exigência foram expressivas, tanto nos modelos brutos (não negros: $R P=2,24$; negros: $\mathrm{RP}=1,83$ ), como nos ajustados (não negros: $\mathrm{RP}=1,91$; negros: $\mathrm{RP}=1,57$ ). 
Tabela 1. Características psicossociais do trabalho segundo raça e gênero. Trabalhadores/as de saúde. Bahia (2012)

\begin{tabular}{|c|c|c|c|c|}
\hline \multirow{2}{*}{ Variáveis } & $\begin{array}{c}\text { Homens } \\
\text { negros }\end{array}$ & $\begin{array}{l}\text { Homens não } \\
\text { negros }\end{array}$ & $\begin{array}{c}\text { Mulheres } \\
\text { negras }\end{array}$ & $\begin{array}{c}\text { Mulheres não } \\
\text { negras }\end{array}$ \\
\hline & $\%$ & $\%$ & $\%$ & $\%$ \\
\hline \multicolumn{5}{|l|}{ Apoio social } \\
\hline Baixo & 29,3 & 27,1 & 71,7 & 69,3 \\
\hline Alto & 70,7 & 72,9 & 28,3 & 30,7 \\
\hline \multicolumn{5}{|c|}{ Demanda psicológica } \\
\hline Baixa & 51,1 & 43,9 & 53,7 & 49,0 \\
\hline Alta & 48,9 & 56,1 & 46,3 & 51,0 \\
\hline \multicolumn{5}{|c|}{ Controle sobre o trabalho } \\
\hline Baixo & 49,3 & 29,9 & 51,6 & 43,4 \\
\hline Alto & 50,7 & 70,1 & 48,4 & 56,6 \\
\hline \multicolumn{5}{|l|}{ Grupos do MDC } \\
\hline Baixa Exigência & 23,8 & 30,6 & 26,6 & 28,9 \\
\hline Trabalho ativo & 26,6 & 39,5 & 21,8 & 27,9 \\
\hline Trabalho passivo & 27,2 & 12,9 & 26,9 & 19,7 \\
\hline Alta exigência & 22,4 & 17,0 & 24,7 & 23,5 \\
\hline
\end{tabular}

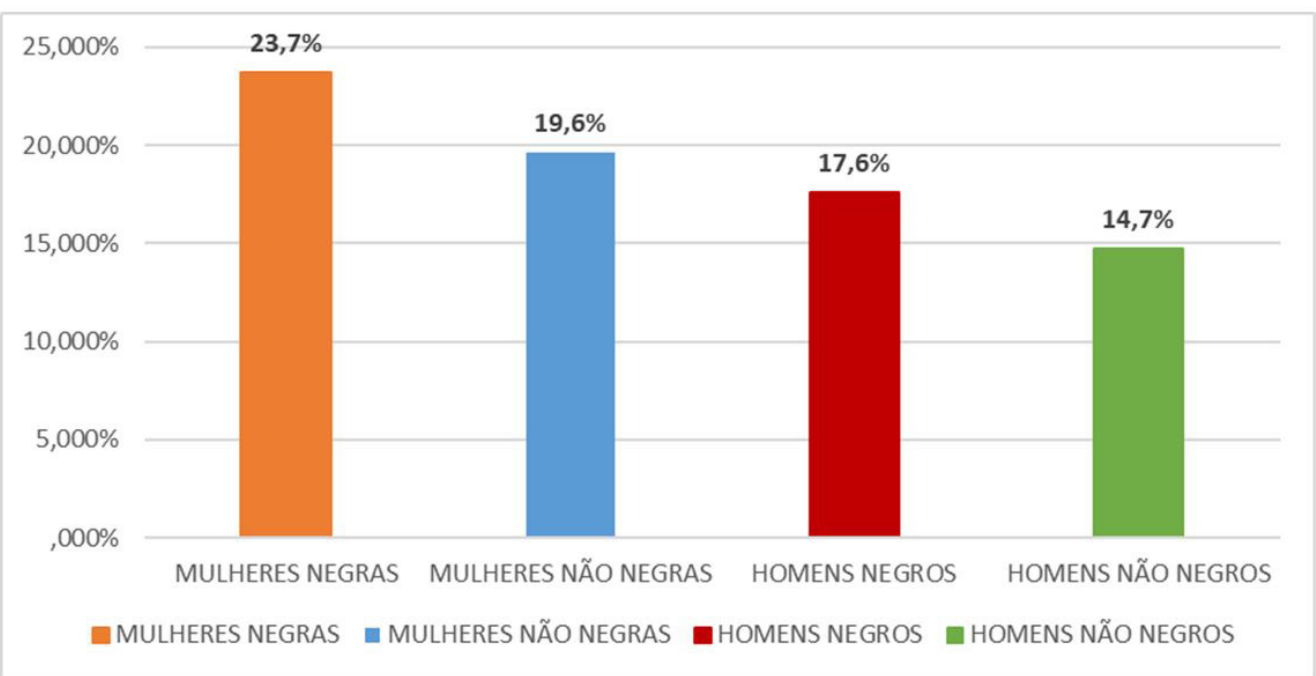

Figura 1. Distribuição das prevalências (\%) de TMC, segundo raça e gênero. Trabalhadores/as de saúde. Bahia (2012)

Os modelos finais obtidos foram analisados quanto à adequação dos dados. A curva ROC permitiu a análise diagnóstica por meio de averiguação dos níveis de sensibilidade e especificidade, obtendo-se área igual a 0,65 para homens negros, 0,71 para homens não negros, 0,62 para mulheres negras e 0,71 para mulheres não negras, evidenciando bom desempenho geral dos modelos. 
Tabela 2. Prevalência, razões de prevalência e respectivos intervalos de confiança de transtornos mentais comuns (TMC) segundo estressores ocupacionais estratificados por raça e gênero (análise bruta). Trabalhadores/as de saúde. Bahia (2012)

\section{TMC}

\begin{tabular}{|c|c|c|c|c|c|c|c|c|}
\hline \multirow{2}{*}{ Variáveis } & \multicolumn{2}{|c|}{ Homens negros } & \multicolumn{2}{|c|}{ Homens não negros } & \multicolumn{2}{|c|}{ Mulheres negras } & \multicolumn{2}{|c|}{ Mulheres não negras } \\
\hline & $\mathbf{P}(\%)$ & RP (IC 95\%) & $\mathbf{P}(\%)$ & RP (IC 95\%) & $\mathbf{P}(\%)$ & RP (IC 95\%) & $\mathbf{P}(\%)$ & RP (IC 95\%) \\
\hline \multicolumn{9}{|l|}{ Apoio Social } \\
\hline Baixo & 18,5 & $0,97(0,64-1,49)$ & 14,6 & $0,56(0,26-1,20)$ & 23,9 & $1,08(0,89-1,31)$ & 21,4 & $1,37(0,84-2,23)$ \\
\hline Alto & 18,9 & --- & 25,7 & --- & 22,0 & --- & 15,5 & --- \\
\hline \multicolumn{9}{|c|}{ Demanda psicológica } \\
\hline Baixa & 13,8 & --- & 12,1 & --- & 18,6 & --- & 12,7 & --- \\
\hline Alta & 21,3 & $1,54(1,03-2,3)^{*}$ & 16,7 & $1,37(0,61-3,08)$ & 29,6 & $1,59(1,34-1,87)^{*}$ & 25,1 & $1,98(1,29-3,03)^{*}$ \\
\hline \multicolumn{9}{|c|}{ Controle sobre o trabalho } \\
\hline Baixo & 19,4 & $1,15(0,78-1,70)$ & 18,6 & $1,41(0,63-3,16)$ & 26,9 & $1,35(1,14-1,60)^{*}$ & 27,2 & $1,79(1,21-2,65)^{*}$ \\
\hline Alto & 16,7 & --- & 13,1 & --- & 19,9 & --- & 15,2 & --- \\
\hline \multicolumn{9}{|c|}{ Modelo demanda-controle } \\
\hline Baixa exigência & 15,0 & --- & 9,3 & --- & 15,0 & --- & 9,2 & --- \\
\hline Trabalho ativo & 17,2 & $1,14(0,63-2,05)$ & 16,1 & $1,72(0,57-5,23)$ & 25,9 & $1,72(1,31-2,26)^{*}$ & 20,7 & $2,24(1,14-4,38)^{*}$ \\
\hline Trabalho passivo & 12,8 & $0,85(0,45-1,60)$ & 15,8 & $1,69(0,42-6,85)$ & 21,8 & $1,45(1,10-1,91)^{*}$ & 19,2 & $2,08(1,00-4,29)$ \\
\hline Alta exigência & 27,6 & $1,83(1,07-3,13)^{*}$ & 20,8 & $2,24(0,66-7,55)$ & 32,4 & $2,16(1,67-2,78)^{*}$ & 32,3 & $3,49(1,84-6,58)^{*}$ \\
\hline
\end{tabular}

*Resultados que obtiveram valor de $\mathrm{p} \leq 0,05$; P: Prevalência. RP: Razão de prevalência. IC: Intervalo de confiança

Tabela 3. Resultados do modelo final de análise da associação de TMC e variáveis de interesse por grupos de gênero e raça. Trabalhadores/as de saúde. Bahia (2012)

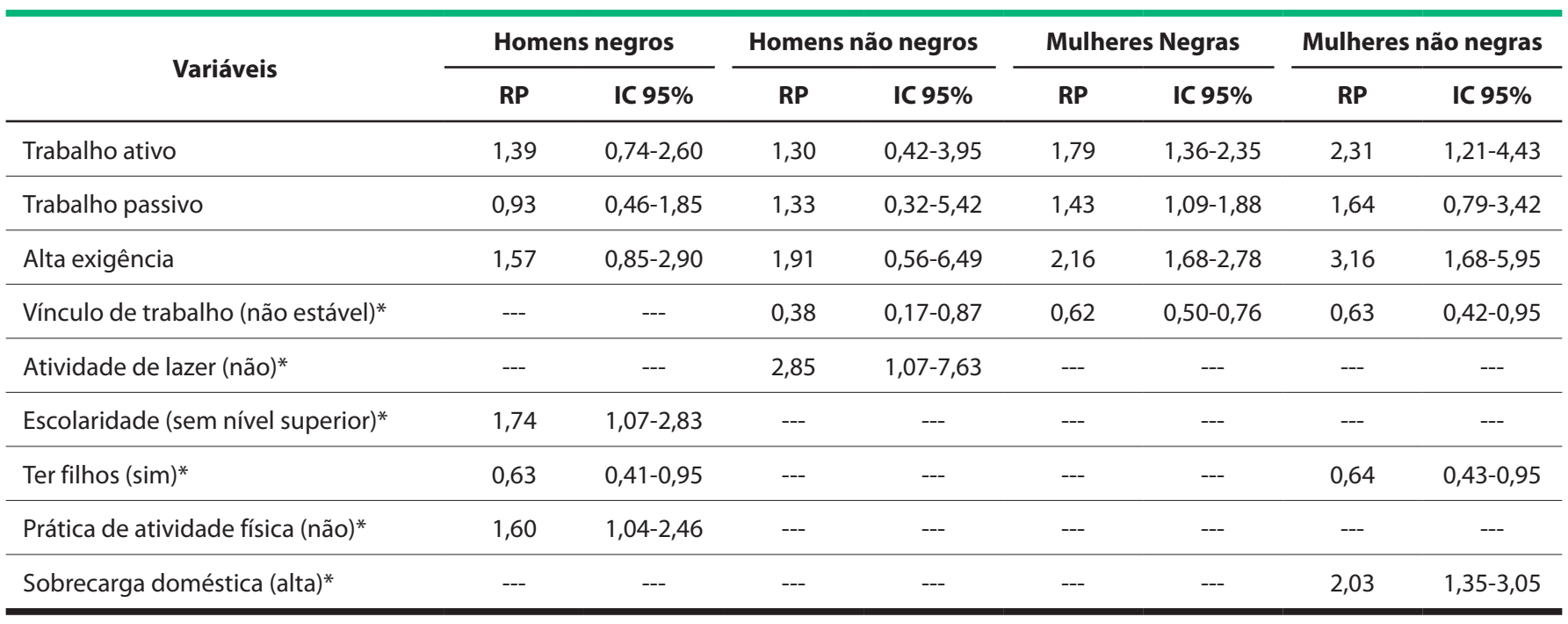

*Variáveis de ajuste que permaneceram no modelo final por grupo de gênero e raça. Entre os homens negros: escolaridade, ter filhos e prática de atividade física; entre os não negros: vínculo de trabalho e atividade de lazer. Entre as mulheres negras: vínculo de trabalho; entre as não negras: vínculo, ter filhos e sobrecarga doméstica RP: Razão de prevalência. IC: Intervalo de confiança

\section{DISCUSSÃO}

Este estudo apontou maior prevalência de TMC entre as mulheres negras, seguidas das mulheres não negras, homens negros e homens não negros, corroborando com outras 
pesquisas que encontraram tendência similar na prevalência de TMC, segundo grupos de raça e gênero ${ }^{8,16}$.

Dados de literatura evidenciaram maior frequência de adoecimento mental entre as mulheres, sendo observadas altas prevalências de TMC entre as trabalhadoras de saúde em comparação com os homens, variando de $25,0 \%$ a $32,5 \%$ entre as mulheres, e de $9,0 \%$ a $12,2 \%$ em homens ${ }^{2,17,18}$. Quanto à raça, os poucos estudos existentes com trabalhadores evidenciaram que a população negra apresenta maior prevalência de TMC com relação à população não negra ${ }^{16,19}$. Destaca-se ainda que o estudo que avaliou interação entre raça e gênero encontrou resultados que fortalecem a hipótese de que esses fatores, em conjunto, elevam a ocorrência de TMC ${ }^{2}$.

A associação de TMC com gênero, com maior ocorrência entre as mulheres, pode ser entendida em função de responsabilidades assumidas com a família e cuidados dos filhos, longas jornadas de trabalho, inserção em postos de trabalho mais precários e menos valorizados, somando-se as atividades domésticas e as atividades laborais, como tem sido apontado na literatura ${ }^{7,8}$. Assim, ao mesmo tempo, a mulher é exposta à sobrecarga laboral e a possibilidades reduzidas de atividades de lazer e de cuidado próprio. Isto, por sua vez, pode contribuir para acúmulo de ansiedade, desgaste, estresse e transtornos mentais ${ }^{20}$.

Observou-se maior frequência de adoecimento mental na população (entre os homens, nos negros; entre as mulheres, nas negras). Diversos estudos observaram resultados similares $2,8,16,19$, corroborando a hipótese de que a raça é um marcador de desigualdades com influência na ocorrência de adoecimento mental, aumentando a vulnerabilidade de alguns grupos. Assim, as desigualdades estabelecidas podem influenciar na saúde mental, incrementando as prevalências de TMC entre negras e negros ${ }^{1}$.

Os modelos finais de análise evidenciaram diferenças na relação entre TMC e estressores ocupacionais nos grupos, como também mostraram relevância de covariáveis específicas nesses grupos. Observe-se que as variáveis retidas nos modelos finais, como potenciais confundidores, foram diferentes nos grupos. Portanto, o estudo identificou aspectos distintos que devem ser mais bem conhecidos em explorações futuras, bem como orientar potenciais intervenções em cada um desses grupos. Assim, apesar de este estudo dedicar-se à análise específica de estressores ocupacionais e TMC, a observação de diferentes características associadas ao adoecimento psíquico nos incita a compreender um pouco melhor esses achados, com análise mais pormenorizada desses resultados.

No modelo final obtido entre os negros, não ter nível superior e não realizar atividade física elevaram a prevalência de TMC, enquanto ter filhos reduziu a prevalência. Diversos estudos apontam que quanto maior escolaridade, melhores são as oportunidades, o que influencia nas condições socioeconômicas, nas características do trabalho e na inserção na sociedade. Assim, menores níveis de escolaridade podem implicar em redução das oportunidades de acesso ao mercado de trabalho e em relações de trabalho mais precárias e mal remuneradas, aspectos que podem estruturar ou ampliar as diferenças sociais ${ }^{21}$. Cabe lembrar que o setor saúde é majoritariamente feminino e, portanto, os postos de trabalho disponíveis para os homens podem ser restritos. Logo, níveis menores de escolaridade podem definir acesso apenas a ocupações mais precárias, desvalorizadas e demandantes. Isto pode explicar em parte a razão pela qual este aspecto pareceu mais contundente entre os negros. A relevância das atividades físicas - aumentando a prevalência de TMC - entre os homens negros pode decorrer de jornadas de trabalho longas e desgastantes, que reduzem o tempo livre para outras atividades; do alto nível de tensão; das questões culturais relacionadas a importância ou não da atividade física, e das condições socioeconômicas, vivenciadas pelos homens negros. Resultados similares foram encontrados em outros estudos ${ }^{22,23}$.

Ter filhos associou-se à menor prevalência de TMC entre os homens negros e mulheres não negras. Diversos estudos destacam que as mulheres estão mais expostas ao adoecimento mental quando possuem filhos, em função das diferenças na construção social dos papéis entre homens e mulheres - que atribui às mulheres a responsabilidade de cuidado - e da dupla jornada de trabalho ${ }^{20}$. No entanto, os dados encontrados neste estudo evidenciaram que ter filhos, entre as mulheres não negras, é um fator que reduz o adoecimento mental. É possível 
que essas mulheres, que apresentaram níveis de escolaridade mais elevados, possam ter posições ocupacionais mais destacadas, com melhores salários, que as possibilite constituir uma rede de apoio de cuidados aos filhos, evitando sobrecarga de atribuições. Esse resultado chama a atenção para o caráter múltiplo das relações que encerram afeto e envolvimento emocional - como as mantidas com a família - e nos alerta sobre a necessidade de investigar mais detidamente essas vivências, sem adotar um ponto inicial unidirecional apontado nas análises mais frequentes no campo.

Vínculo de trabalho não estável associou-se aos TMC entre os homens não negros e entre as mulheres (negras e não negras). Esse achado apontou na direção oposta ao esperado segundo dados de literatura, que destacam os vínculos estáveis como mais protegidos do que os instáveis (temporários/não concursados), nos quais predominam a insegurança e a precariedade. Assim, este é um dado que precisa ser melhor compreendido. Na área da saúde, as características dos vínculos de trabalho têm se transformado profundamente. Na população estudada, o vínculo de trabalho estável predominou entre os homens negros e entre as mulheres negras e não negras. Assim, observa-se que algumas profissões, a exemplo dos agentes de saúde, que também foi predominante entre esses grupos, tendem a ser, por lei, ocupados por profissionais concursados. Apesar da estabilidade do vínculo, esses postos de trabalho têm sido precários, com baixos salários e desvalorização profissional e social, fatores estes associados ao adoecimento mental ${ }^{6}$. As relações entre emprego e a conformação dos vínculos de trabalho na saúde é de grande complexidade e, assim, percebe-se que possuir vínculo de trabalho estável parece não ser suficiente para garantir as melhores condições de saúde.

Achados relativos à associação de prevalência de TMC e ausência de prática de atividades de lazer, observada entre os homens não negros, é coerente com resultados de outros estudos em que a prática de atividades de lazer favoreceu a redução da ansiedade, estresse e dos transtornos mentais, propiciando bem-estar e prazer ${ }^{23,24}$. Similarmente, a associação de alta sobrecarga doméstica e maior ocorrência de TMC, encontrada aqui entre as mulheres não negras, também corrobora dados de literatura que apontam a sobrecarga de trabalho como fator desencadeador de desgaste físico e mental ${ }^{25,26}$.

Com relação ao estresse ocupacional eTMC, foco deste estudo, os resultados evidenciaram que os estressores do trabalho analisados - baixo controle sobre o próprio trabalho e alta demanda psicológica - se associaram positivamente com TMC entre as mulheres negras (alta exigência, trabalho passivo e trabalho ativo) e não negras (alta exigência e trabalho ativo), corroborando o modelo proposto por Karasek (1979), no pressuposto de que níveis elevados de demanda psicológica e baixo controle sobre o trabalho exercido configuram-se situação de risco ao adoecimento físico e mental ${ }^{6,7,19,24}$.

A exposição a essas duas dimensões psicossociais nocivas simultaneamente - alta demanda e baixo controle - estava mais fortemente associada aos TMC do que quando expostas a apenas uma dessas dimensões ou ao grupo de referência, corroborando com outros achados ${ }^{19,27}$. O trabalho em alta exigência pode acarretar sofrimento psicológico, como fadiga, estresse e depressão. Esse desgaste ocorre quando o trabalhador não consegue realizar suas atividades adequadamente em função do pouco controle sobre as circunstâncias ambientais ${ }^{28}$. Além de elevar a frequência de TMC, como evidenciou o estudo, a exposição prolongada a esse nível de estresse tende a provocar outras morbidades, como doenças cardiovasculares, musculoesqueléticas, cefaleias, entre outros desfechos sobre a saúde ${ }^{29}$.

Em relação aos níveis intermediários de estresse ocupacional, o trabalho ativo foi mais relevante para a prevalência de TMC do que o trabalho passivo, resultado este similar ao encontrado em outros estudos $s^{6,7,19,30}$. Segundo pressupostos do MDC, apesar das exigências elevadas neste grupo, o controle sobre as tarefas também é alto, o que possibilita aprendizado e crescimento do trabalhador ${ }^{31}$, que são condições positivas do trabalho. Entretanto, neste estudo, observou-se que trabalho ativo se associou aos TMC entre as mulheres (negras e não negras). $O$ alto controle indica uma tentativa compensatória de efeitos nocivos provenientes das altas demandas ${ }^{5}$. No entanto, pode ser que, em situações de demandas elevadas, que são frequentes no setor saúde, esse efeito de compensação do controle não seja efetivo. Ou seja, se as demandas são muito elevadas, o controle pode perder efeito moderador dos efeitos 
negativos associados. Esse resultado reforça o fato de que as demandas do trabalho, neste grupo, exercem papel determinante para os agravos na saúde mental.

Um importante marcador de gênero foi observado nas relações entre estresse ocupacional e ocorrência dos TMC: essa associação somente foi identificada, com bases nos critérios estatísticos adotados, entre as mulheres. Destaca-se que, entre as mulheres negras, todas as situações de exposição do MDC estiveram associadas à ocorrência de TMC. Esse dado pode ser justificado pelas desigualdades relacionadas à raça e ao gênero, vividas pelas mulheres e principalmente pelas negras, as quais é sabido que se encontram em situação de amplas desvantagens com relação aos postos e oportunidades de trabalho, às características e condições nas quais realiza o trabalho e aos processos de valorização profissional, fatores que podem ocasionar maior estresse e maior adoecimento ${ }^{32}$.

Os dados relativos aos estressores ocupacionais e TMC entre os homens merecem maior aprofundamento. Mesmo as dimensões do modelo demanda-controle não tendo alcançado significância estatística no modelo final de análise para os homens (negros e não negros), observou-se que as razões de prevalência encontradas foram elevadas. A possibilidade de insuficiência do tamanho amostral para apreender tal relação, por exemplo, deve ser considerada. Como se sabe, no trabalho de saúde, há ampla predominância das mulheres, portanto a participação masculina é reduzida, afetando o tamanho amostral entre os homens. Logo, embora o tamanho da amostra seja relativamente grande, quando dividido pelos estratos de raça e gênero, alguns grupos, como o dos homens não negros, ficaram com números pequenos e, consequentemente, perde-se poder na análise pretendida. O reduzido tamanho amostral desses subgrupos na população pode ter inviabilizado a observância estatisticamente significante de diferenças nesses grupos. Portanto, a não observância de significância estatística deve ser interpretada com cautela, não sendo possível descartar associação desses estressores ocupacionais com TMC, entre os homens.

Cabe mencionar a ausência de base comparativa com relação a estudos que abordassem os estressores ocupacionais e transtornos mentais comuns, segundo estratos de gênero e raça. Como esse tema de estudo ainda é pouco explorado, a comparação dos resultados obtidos neste estudo com resultados de outras pesquisas foi limitada. Devem-se considerar ainda as limitações inerentes aos estudos transversais, a exemplo da causalidade reversa e do efeito do trabalhador sadio como importantes limites na análise dos resultados obtidos.

Apesar dessas limitações, este estudo aporta importantes subsídios para os campos da saúde e do trabalho, evidenciando diferenças entre os grupos estudados e jogando luz sobre especificidades que precisam ser exploradas e mais bem compreendidas, o que fomenta novos estudos nessa perspectiva.

Os dados encontrados revelaram que existem diferenças quando se trata de raça e gênero na relação entre estressores ocupacionais e TMC. Os homens negros e não negros apresentaram menores prevalências de TMC. Entre as mulheres negras e não negras, os resultados apontaram uma discreta diferença na prevalência de TMC, com as mulheres negras apresentando prevalência maior do que as não negras. As mulheres, independentemente da raça, vivenciavam situações de maior vulnerabilidade para os TMC com relação aos homens. As desigualdades raciais associaram-se, assim, às vulnerabilidades de gênero, potencializando efeitos nocivos à saúde. As mulheres negras constituíram o grupo de maior vulnerabilidade para os transtornos mentais, sendo aquelas que também acumularam as maiores desigualdades no conjunto de variáveis investigadas. Em síntese, os resultados revelam a importância de fomentar essa linha de investigação, com novos e contínuos investimentos, e foco nas identidades sociais e nas desigualdades em saúde, verificando como estas podem afetar a saúde mental dos trabalhadores.

Esta pesquisa permitiu o conhecimento das situações de trabalho associadas com TMC, possibilitando identificar os grupos mais expostos ao estresse ocupacional e aos seus efeitos na saúde mental. Assim, as altas prevalências encontradas mostram a necessidade de estabelecer medidas no sentido de melhorar as condições de trabalho desses trabalhadores e trabalhadoras de maneira equânime, no intuito de reduzir as desigualdades de gênero e raça. 
É necessário propor estratégias que possam promover a saúde, melhorias nas condições e no ambiente de trabalho, bem como a implementação de políticas públicas no monitoramento e controle dos aspectos psicossociais no trabalho, tendo um olhar diferenciado para mulheres, em especial para as mulheres negras, que apresentaram maior prevalência da doença.

\section{REFERÊNCIAS}

1. Pascoe EA, Smart Richman L. Perceived discrimination and health: a meta-analyitic review. Psychol Bull. 2009;135(4):531-54. http://dx.doi.org/10.1037/a0016059. PMid:19586161.

2. Smolen JR, Araújo EM, Oliveira NF, Araújo TM. Intersectionality of race, gender, and common mental disorders in Northeastern Brazil. Ethn Dis. 2018;28(3):207-14. http://dx.doi.org/10.18865/ed.28.3.207.

3. Araújo EM, Costa MCN, Hogan VK, Araújo TM, Dias AB, Oliveira LOA. A utilização da variável raça/cor em Saúde Pública: possibilidades e limites. Interface - Comunic., Saude. Educ. 2009;13(31):383-94. http:// dx.doi.org/10.1590/S1414-32832009000400012.

4. Silva VC, Silva WS. Marcadores sociais da diferença: uma perspectiva interseccional sobre ser estudante negro e deficiente no Ensino Superior brasileiro. Rev Bras Educ Espec. 2018;31(62):569-85. http://dx.doi. org/10.5902/1984686X30948.

5. Marcelino A Fo, Araújo TM. Estresse ocupacional e saúde mental dos profissionais do centro de especialidades médicas de Aracaju. Trab Educ Saúde. 2015;13(Suppl 1):177-99. http://dx.doi. org/10.1590/1981-7746-sip00016.

6. Braga LC, Carvalho LR, Binder MCP. Condições de trabalho e transtornos mentais comuns em trabalhadores da rede básica de saúde de Botucatu (SP). Ciênc. saúde coletiva. 2010;15(Suppl 1):1585-96. http://dx.doi. org/10.1590/S1413-81232010000700070.

7. Araújo TM, Graça CC, Araújo E. Estresse ocupacional e saúde: contribuições do Modelo Demanda-Controle. Ciênc. saúde coletiva. 2003;8(4):991-1003. http://dx.doi.org/10.1590/S1413-81232003000400021.

8. Smolen JR, Araújo EM. Raça/cor da pele e transtornos mentais no Brasil: uma revisão sistemática. Ciênc. saúde coletiva. 2017;22(12):4021-30. http://dx.doi.org/10.1590/1413-812320172212.19782016.

9. Bowleg L.The Problem with the phrase women and minorities: intersectionality - an important theoretical framework for public health. Am J Public Health. 2012;102(7):1267-73. http://dx.doi.org/10.2105/ AJPH.2012.300750. PMid:22594719.

10. Santos KOB, Araújo TM, Pinho PS, Silva ACC. Avaliação de um instrumento de mensuração de morbidade psíquica: estudo de validação do self-reporting questionnaire (SRQ-20) [Internet]. Rev Baiana Saúde Publica. 2010;34(3):544-60 [citado em 2018 Nov 16]. Disponível em: http://files.bvs.br/upload/S/0100-0233/2010/ v34n3/a1881.pdf

11. Karasek R. Job demand, job decision latitude, and mental strain: implications for job redesign. Adm Sci Q. 1979;24(2):285-308. http://dx.doi.org/10.2307/2392498.

12. Araújo TM, Karasek R. Validity and reliability of the job content questionnaire in formal and informal jobs in Brazil. Scand J Work Environ Health. 2008;34(Suppl 6):52-9.

13. Aquino EML. Gênero, trabalho e hipertensão arterial: um estudo de trabalhadores de enfermagem em Salvador, Bahia [tese]. Salvador: Instituto de Saúde Coletiva, Universidade Federal da Bahia; 1996.

14. Hosmer DW, Lemeshow S. Applied logistic regression. Boca Raton: John Wiley and Sons; 2000. http:// dx.doi.org/10.1002/0471722146.

15. Coutinho LMS, Scazufca M, Menezes PR. Métodos para estimar razão de prevalência em estudos de corte transversal. Rev Saude Publica. 2008 dez;42(6):992-8. http://dx.doi.org/10.1590/S003489102008000600003. PMid:19009156.

16. Anselmi L, Barros FC, Minten GC, Gigante DP, Horta BL, Victora CG. Prevalência e determinantes precoces dos transtornos mentais comuns na coorte de nascimentos de 1982, Pelotas, RS. Rev Saude Publica. 2008 dez;42(Suppl 2):26-33. http://dx.doi.org/10.1590/S0034-89102008000900005. PMid:19142342.

17. Alves AP, Pedrosa LAK, Coimbra MAR, Miranzi MAS, Hass VJ. Prevalência de transtornos mentais comuns entre profissionais de saúde. Rev. enferm UERJ. 2015;23(1):64-9. http://dx.doi.org/10.12957/ reuerj.2015.8150.

18. Carvalho DB, Araújo TM, Bernardes KO. Transtornos mentais comuns em trabalhadores da Atenção Básica à Saúde. Rev Bras Saúde Ocup. 2016;41(17):e17. http://dx.doi.org/10.1590/2317-6369000115915.

19. Farias MD, Araújo TM. Transtornos mentais comuns entre trabalhadores da zona urbana de Feira de SantanaBA. Rev Bras Saúde Ocup. 2011;36(123):25-39. http://dx.doi.org/10.1590/S0303-76572011000100004. 
20. Lucchese R, Sousa K, Bonfin SP, Vera I, Santana FR. BSP, Vera I, Santana FR. Prevalência de transtorno mental comum na atenção primária. Acta Paul Enferm. 2014;27(3):200-7. http://dx.doi.org/10.1590/19820194201400035.

21. Ludermir AB, Melo Filho DA. Condições de vida e estrutura ocupacional associadas a transtornos mentais comuns. Rev Saude Publica. 2002;36(2):213-21. http://dx.doi.org/10.1590/S0034-89102002000200014. PMid:12045803.

22. Oliveira AMN, Araújo TM. Situações de desequilíbrio entre esforço-recompensa e transtornos mentais comuns em trabalhadores da atenção básica de saúde. Trab Educ Saúde. 2018;16(1):243-62. http://dx.doi. org/10.1590/1981-7746-sol00100.

23. Rocha SV, Almeida MMG, Araújo TM, Virtuoso JS Jr. Atividade física no lazer e transtornos mentais comuns entre idosos residentes em um município do nordeste do Brasil. J Bras Psiquiatr. 2011;60(2):80-5. http:// dx.doi.org/10.1590/S0047-20852011000200002.

24. Araújo TM, Aquino E, Menezes G, Santos CO, Aguiar L. AE, Menezes G, Santos CO, Aguiar L. Aspectos psicossociais do trabalho e distúrbios psíquicos entre trabalhadoras de enfermagem. Rev Saude Publica. 2003;37(4):424-33. http://dx.doi.org/10.1590/S0034-89102003000400006. PMid:12937702.

25. Pinho PS, Araújo TM. Associação entre sobrecarga doméstica e transtornos mentais comuns em mulheres. Rev Bras Epidemiol. 2012 set;15(3):560-72. http://dx.doi.org/10.1590/S1415-790X2012000300010. PMid:23090303.

26. Araújo TM, Pinho PS, Almeida MMG. Prevalência de transtornos mentais comuns em mulheres e sua relação com as características sociodemográficas e o trabalho doméstico. Rev Bras Saúde Mater Infant. 2005;5(3):337-48. http://dx.doi.org/10.1590/S1519-38292005000300010.

27. Kirchhof ALC, Magnago TSBS, Camponogara S, Griep RH, Tavares JP, Prestes FC, et al. Condições de trabalho e características sócio-demográficas relacionadas à presença de distúrbios psíquicos menores em trabalhadores de enfermagem. Texto Contexto Enferm. 2009;18(2):215-23. http://dx.doi.org/10.1590/ S0104-07072009000200003.

28. Alves MGM, Hokerberg YHM, Faerstein E. Tendências e diversidade na utilização empírica do Modelo Demanda-Controle de Karasek (estresse no trabalho): uma revisão sistemática. Rev Bras Epidemiol. 2013;16(1):125-36. http://dx.doi.org/10.1590/S1415-790X2013000100012. PMid:23681329.

29. Shimabuku RH, Mendonca H, Fidelis A. Presenteísmo: contribuições do Modelo Demanda-Controle para a compreensão do fenômeno. Cad Psicol Soc Trab. 2017;20(1):65-78. http://dx.doi.org/10.11606/issn.19810490.v20i1 p65-78.

30. Araújo TM, Mattos AIS, Almeida MMG, Santos KOB. Aspectos psicossociais do trabalho e transtornos mentais comuns entre trabalhadores da saúde: contribuições da análise de modelos combinados. Rev Bras Epidemiol. 2016;19(3):645-57. http://dx.doi.org/10.1590/1980-5497201600030014. PMid:27849277.

31. Jacinto A, Tolfo SR. Fatores psicossociais de risco no trabalho e Transtorno Mental Comum: uma revisão sistemática de estudos que utilizaram os instrumentos JCQ, JSS e SRQ-20. Rev. Psicol. IMED. 2017;9(2):10724. http://dx.doi.org/10.18256/2175-5027.2017.v9i2.1432.

32. Carlotto MS, Barcinski M, Fonseca R. Transtornos mentais comuns e associação com variáveis sociodemográficas e estressores ocupacionais: uma análise de gênero. Estud Pesqui Psicol. 2015;15(3):100626. http://dx.doi.org/10.12957/epp.2015.19424. 